\title{
There's the Rub: a novel ubiquitin-like modification linked to cell cycle regulation
}

\author{
Mark Hochstrasser ${ }^{1}$ \\ Department of Biochemistry and M olecular Biology, The University of Chicago, Chicago, Illinois 60637 USA
}

Covalent attachment of the polypeptide ubiquitin to intracellular proteins is achieved through an intricate and highly conserved enzymatic pathway that is beginning to be understood in some detail (for review, see Wilkinson 1995; Hochstrasser 1996; Pickart 1997). U bi quitin is joined reversibly to proteins by an isopeptide linkage of the carboxy-terminal carboxyl group of ubiquitin to lysine side chains of the acceptor proteins (Fig. 1). In the majority of cases examined, the modified protein, particularly when it is attached to multiple ubiquitin molecules, is targeted to a large, abundant intracellular protease called the proteasome, which degrades the substrate into small peptides but allows recycling of the ubiquitin moieties.

Over the past few years, it has emerged that eukaryotes also express a set of ubiquitin-like proteins (U bls) that are significantly diverged from ubiquitin itself yet are also ligated to other proteins (Haas and Siepmann 1997; Johnson and Hochstrasser 1997). The reactions involving these variants appear to have much in common with those of ubiquitin, but the U bls have novel regulatory functions not necessarily linked to proteolysis. In this issue, Lammer et al. describe an unexpected connection between a Ubl called Rubl (related to ubiquitin 1) from the yeast Saccharomyces cerevisiae and a ubi quitin-protein ligase complex that is critical for progression from $G_{1}$ to $S$ phase in the cell cycle. In this commentary, I will discuss these new results together with recent related devel opments on the mechanisms and functions of U bl-protein ligation.

\section{Rubl and NEDD8: new Ubl modifiers}

A distinctive feature of ubi quitin is that it is al ways synthesized in precursor form, with one or more amino acids following a Gly-Gly dipeptide that forms the carboxyl terminus of the mature protein (Fig. 2A). These "tail" sequences are clipped off by members of a diverse group of enzymes called deubiquitinating enzymes. Analogous attributes turn out to be a hallmark of the subset of $U$ bls that also form covalent adducts with other proteins (Fig. 2A). In contrast, many Ubls have

1E-MAIL hoc1@midway.uchicago.edu; FAX (773) 702-0439. nonremovable ubiquitin-like domains, and these domains are invariably missing the Gly-Gly element.

Several years ago, the yeast genome project identified a novel DNA sequence with the potential for encoding a $77-$ residue protein that is $53 \%$ identical ( $72 \%$ similar) to ubiquitin (Hochstrasser 1996). Interestingly, the product of this gene, dubbed RUB1, is even more closely related (59\% i dentity) to a protein encoded by a devel opmentally regulated gene from mammals called NEDD8 (Kumar et al. 1993) (Fig. 2A). The predicted translation products of these genes include a Gly-Gly dipepti de followed by one or five additional residues, suggesting that these polypeptides might be conjugated to other proteins. Late last year it was reported that N EDD8 is carboxy-terminally processed and becomes coval ently coupl ed to at least one cellular protein (Kamitani et al. 1997). The same has now been shown to be true for Rubl in yeast (Lammer et al. 1998; Liakopoulos et al. 1998). A small number of Rub1protein conjugates is readily detectable by immunoblot analysis, one of the most prominent involving, surprisingly, Cdc53, a protein that functions in ubiquitin-protein ligation. As will become clear below, Rub1 and NEDD 8 are almost certainly functional homologs, and Rubl/NEDD8 ligation to proteins is likely to have important growth regulatory roles in plants, mammals, and presumably many other organisms.

The sequence differences between Rubl and ubi quitin are clustered when viewed on the three-dimensional structure of ubiquitin, leaving the hydrophobic face of ubiquitin largely intact. Lysine residues at positions 29 and 48 are al so conserved. These two lysines have both been shown to participate in polyubiquitin chain formation in yeast cells, and Lys-48 and the hydrophobic surface patch of ubiquitin have been implicated in substrate targeting to the proteasome (Pickart 1997). Based on these considerations, it is possible that modification by Rubl can regulate the ubiquitination and/or degradation of at least some cellular proteins. Such conjugation might negatively regulate protein degradation by the proteasome if Rubl attachment competed with ubiquitin conjugation but was less effective in proteasome targeting. Conversely, Rubl might act as an enhancer of proteolysis if protein modification by Rub1 [poly(Rub1)?] were an efficient proteasome-targeting mechanism. 


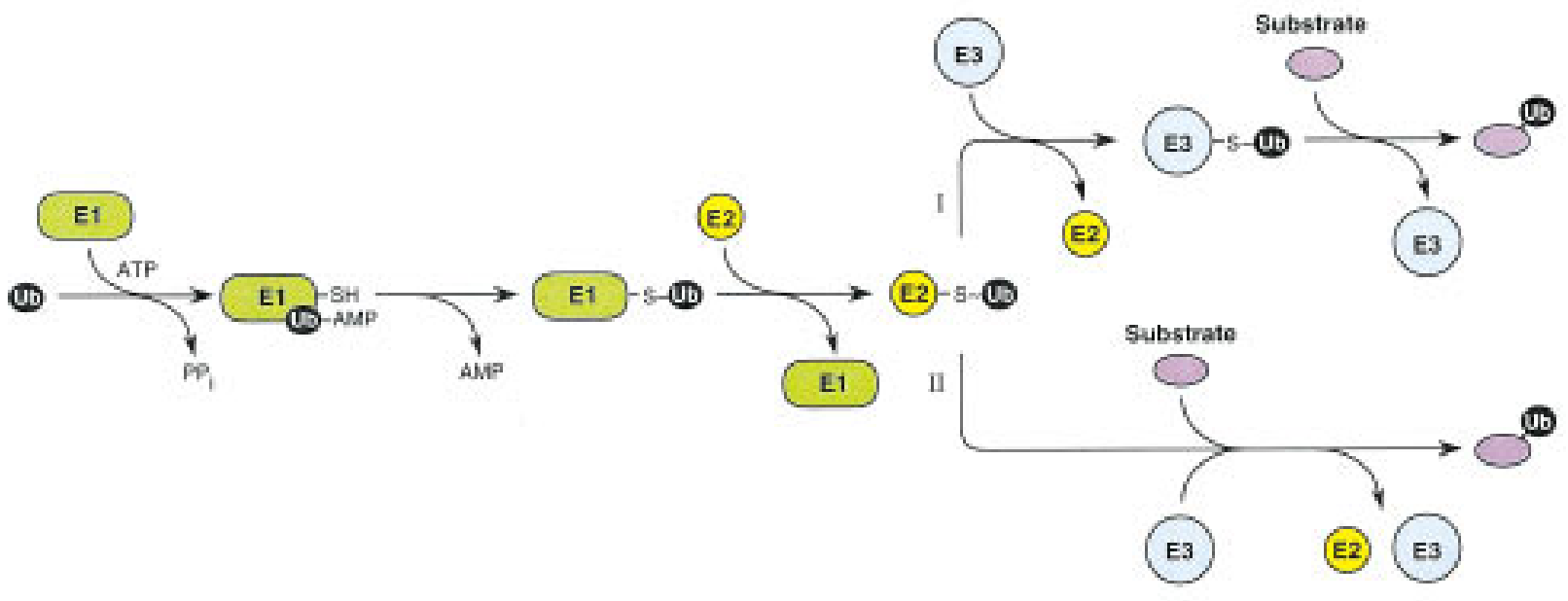

Figure 1. The ubiquitin-protein conjugation pathway. The ligation mechanisms of Rub1/NEDD 8 and other U bls are likely to be very similar. In the first step of the ubiquitin pathway, the carboxy-terminal carboxyl group of ubiquitin (Ub) is activated by E1 (Ubal in yeast) by formation of a mixed anhydride with AMP. The ubiquitin-adenylate is then transferred to a cysteine residue of E1, resulting in a ubiquitin-E1 thiol ester. (A second, tightly bound ubiquitin-adenylate is formed after this step but is not shown here.) Thereafter, the ubiquitin is transferred to a reactive cysteine of a second enzyme, E2 (U bc). Although E2 enzymes can sometimes di rectly catalyze isopeptide bond formation between ubiquitin and substrate protein in vitro, this reaction generally requires the aid of at least one additional factor, called E3 or ubiquitin-protein ligase. Currently, there is evidence for two different E3-based mechanisms for substrate ubiquitination. For at least one structural subclass of E3s (Scheffner et al. 1995), ubiquitin is transferred from the E2 to a thiol group on the E3 protein and only then is conjugated to a lysine side chain of the substrate (pathway I). For other E3s, the E3 appears to function more as an adaptor, juxtaposing the E2-ubiquitin thiolester and substrate to allow transfer of ubiquitin directly from the E2 (pathway II). For the Rub1/N EDD8 and Smt3/SU M O-1 pathways, both E1-like and E2-like enzymes have been identified, but no E3-like activities are known yet.

\section{Ubl-protein ligation: a new twist on an old theme}

All post-translational ubiquitination events begin with the activation of the carboxyl terminus of ubiquitin by the E1 enzyme (Fig. 1; see legend for details). Y east cells have a single E1-encoding gene, UBA 1 , which is essential for viability. For several reasons, E1 is not likely to play a significant role in the selection of ubiquitination targets. Instead, the major determinant of substrate specificity in the ubiquitin conjugation pathway is the E2/E3 ubiquitin-protein ligase complex, which binds the substrate protein and catalyzes isopeptide bond formation with ubiquitin (Figs. 1 and 3). In yeast, 13 genes encoding E2-related enzymes have been identified in the complete genome sequence (Hochstrasser 1996), and both genetic and biochemical data indi cate that different E2s participate in the ubiquitination of distinct substrates. Similarly, a number of structurally diverse E3 factors have been identified, and they are also involved in the degradation of distinct proteins. Significantly, there are now examples both of disparate E2s functioning either together or separately in the modification of individual substrates (Chen et al. 1993) and of the same E2 associating with distinct E3s, which then ubiquitinate different proteins (Skowyra et al. 1997). These observations have led to the idea that the ubiquitin conjugation system can expand its repertoire of substrate specificities through association of a limited set of E2 and E3 proteins into multiple heteromeric complexes (Chen et al. 1993; Skowyra et al. 1997).

With the identification of conjugation-competent pro- teins that are related to but substantially diverged from ubiquitin, renewed interest has been directed toward the $\mathrm{E} 1$ and E1-like enzymes as they may be the primary factors responsible for distinguishing among ubiquitin and its molecular brethren. Consistent with this possibility, many residues not conserved between the $U$ bls and ubiquitin were shown by mutagenesis studies of ubiquitin to be important for El interaction (Burch and Haas 1994). An important advance in this area came from experiments by Johnson et al. (1997) on the activation of the yeast $\mathrm{U}$ bl called Smt3, which is the orthol og of the vertebrate SUMO-1 protein and is involved in passage of yeast cells through the $G_{2} / M$ phase of the cell cycle (see Johnson and Hochstrasser 1997). They purified and characterized an enzyme capable of forming a thi olester with Smt3 in the presence of ATP, and, unexpectedly, the protein was found to be a heterodimer of A osl and U ba2 (Fig. 2B). Remarkably, U ba2 bears clear similarity to the carboxyl terminus of $U$ bal, the yeast $E 1$, whereas Aosl is related to the amino-terminal region of U bal. The Smt3 thiolester is formed with the Uba2 subunit, but both subunits are required for Smt3 activation in vitro and in vivo. Thus, the yeast Smt3-activating enzyme resembles a split version of $\mathrm{Ubal}$.

It turns out that Rub1/N EDD 8 activation is performed by an exactly anal ogous heterodimeric E1-like complex. Using the ability of a fraction from a human HeLa cell Iysate to form an ATP-dependent and DTT-sensitive adduct with radiolabeled NEDD8, Chau and coworkers purified a 110-kD complex that contained 49- and 60-kD subunits (T. Gladysheva, J. Pohl, F. Melandri, and V. 
A

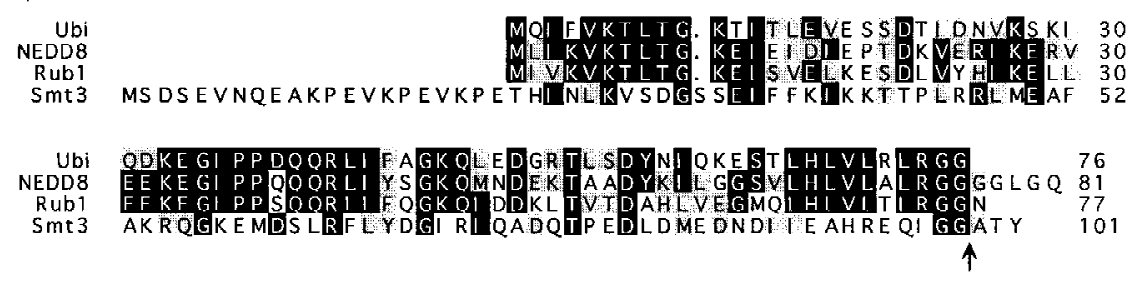

B

Sucsatrate Actioating enzyme

\author{
Ub
}

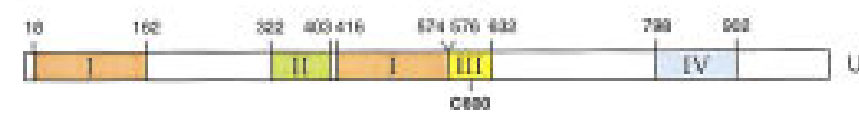

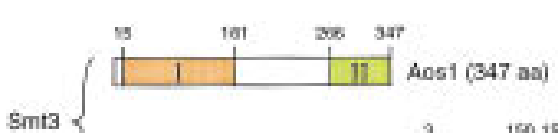
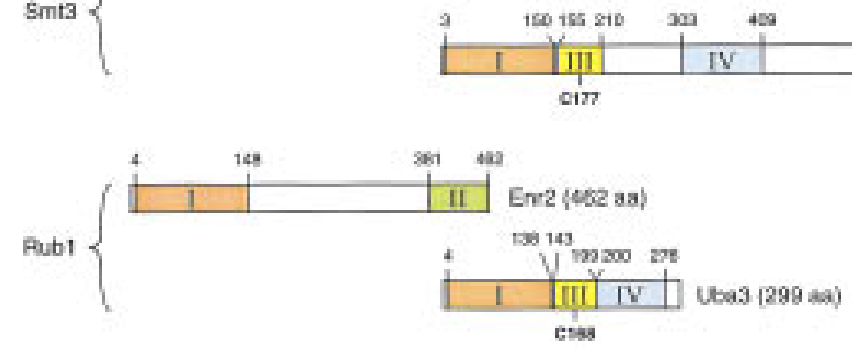

Figure 2. Conjugation-competent Ubls and their specific activating enzymes. (A) Sequence alignment of yeast ubiquitin (mature form) and the precursors of yeast Rubl (PIR accession no. S51867), human N EDD8 (D23662), and yeast Smt3 (U 27233) using the CLUSTALW algorithm. The arrow indicates the site of precursor cleavage. (B) Ubiquitin- and Ubl-activating enzymes. Smt3 is activated by a heterodimer of Aos1 and U ba2, with U ba2 carrying the catalytic Cys residue (in domain III) (Johnson et al. 1997). Rubl activation in yeast requires both Enr2/Ulal and Uba3. A heterodimer of the Enr2 and Uba3 orthologs from human has been observed; the complex is capable of activating NEDD8, the mammalian ortholog of Rub1 (see text). Sequence similarity boxes are those noted by Johnson et al. (1997).
Chau, unpubl.). The 49-kD subunit forms the apparent thiolester with NEDD8, and using microsequence data obtained from this polypeptide, these workers identified a gene that was predicted to encode a protein with sequence similarity to the carboxy-terminal region of Els. The strongest similarity to any yeast gene was to a sequence encoding a putative El-like protein, U ba3 (Hochstrasser 1996), that had been identified as part of the genome sequencing project. Peptides derived from the 60-kD subunit of the HeLa enzyme were identical to a previously identified protein called APP-BP1, which is highly similar to the amino-terminal domain of E1 (Chow et al. 1996). APP-BP1 (see bel ow) was identified originally by its ability to bind to a cytopl asmic segment of the amyloid precursor protein (APP), which is the source of the amyloidogenic peptides that accumulate in patients with Alzheimer's disease.

Independent of the above study, the Jentsch group identified two yeast proteins that together were capable of activating Rubl (Liakopoulos et al . 1998). Based on the analysis of Smt3 activation, they reasoned that a different pair of UBA1-related genes of previously unknown function, UBA3 and ULA1/ENR2, might encode the components of a Rubl-activating complex. U ba3 was shown to form a thiolester with Rubl in vitro but only in the presence of the Enr2 protein. Moreover, deletion of either UBA3 or ENR2 from yeast cells eliminated all detectable Rub1-protein conjugates (at least five distinct species were seen by immunoblot analysis). Lammer et al. (1998) have also demonstrated that one of the subunits, Enr2, is required for Rub1-protein ligation in vivo.
The ENR2 gene product is similar to the product of $A X R 1$, an Arabidopsis thal iana gene that is required in the plant for developmental responses to the hormone auxin. AXR1 served as the starting point for the Estelle group's investigation of the yeast E1-related proteins (Leyser et al. 1993).

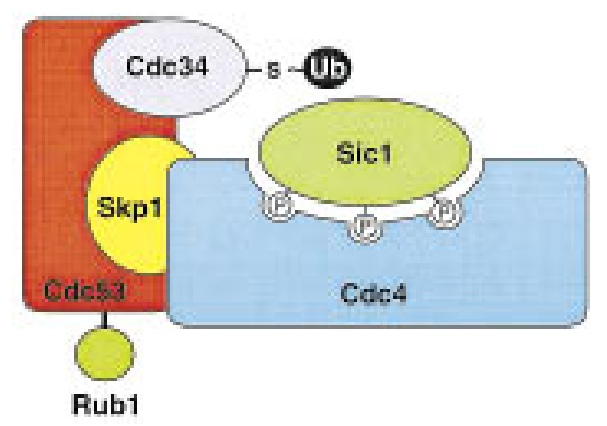

Figure 3. Sicl, a yeast CDK inhibitor, is ubiquitinated by the Cdc34-SCF ${ }^{\mathrm{Cdc} 4}$ complex. SCF ${ }^{\mathrm{Cdc} 4}$ (Skp1-Cdc53-F-box protein Cdc4) is an E3 that contains three different polypeptides. The binding of $\mathrm{Sicl}$ to the $\mathrm{F}$-box protein $\mathrm{Cdc} 4$ requires $\mathrm{Sicl}$ phosphorylation by the $\mathrm{CIn}-\mathrm{Cdc} 28$ kinase. Covalent modification of Cdc53 by Rubl is shown, but whether this form of $\mathrm{Cdc} 53$ is involved in $\mathrm{Sicl}$ degradation is not known. At least in vitro, the modification is unlikely to be absolutely required because the carboxy-terminally truncated Cdc53 protein that is not Rub1modified in vivo is still able to support Sicl ubiquitination in yeast lysates (Feldman et al. 1997) (see text for details). 
An El-like enzyme is not the only conjugation pathway component specific to a particular U bl or ubiquitin. Smt3 and SUMO-1 both can form a thiolester with a specific E2-like protein, U bc9, which does not work with ubiquitin (Desterro et al. 1997; Johnson and Blobel 1997; Schwarz et al. 1998). The same appears to be true for Rub1. Liakopoulos et al. (1998) find that U bc12 is necessary for Rub1-protein conjugation in vivo, and in the presence of Enr2-U ba3, U bc12 can form a thi oester with Rubl in vitro. One might also expect that there are U blspecific E3-like factors, but this remains to be determined.

Lest there remain any doubt about the conservation of the Rub1 and NEDD8 conjugation systems, Estelle and coll eagues have found that A rabidopsis AXR1 (which is $40 \%$ identical to human APP-BP1), when combined in vitro with another protein they call ECR1, results in ECR1 thiolesters with either Rub1 or NEDD8 (M. Estelle, pers. comm.). Similarly, D. Liakopoulos and S. Jentsch (unpubl.) have expressed NEDD8 in yeast and found nearly the same conjugate pattern as with Rubl, including Cdc53; this conjugate profile al so depended on Enr2, U ba3, and U bc12.

In summary, conjugation of proteins to both Smt3/ SUM O-1 and Rub1/NEDD 8 depends on specific E2-like enzymes and a specific heterodimeric activating enzyme complex. This heterodimer organization of the E1-like factors is remarkably well conserved among yeast, plants, and mammals, suggesting that it provides some functional advantage. Conceivably, $U$ bl-protein ligation will involve combinatorial specificity of a sort anal ogous to that discussed earlier for E2s and E3s in ubiquitinprotein ligation, with the new wrinkle that different heterodimeric combinations of E1-related polypeptides (and possibly E2s) may contribute to selection of the appropriate modifier protein.

\section{Rubl and the regulation of cell cycle progression}

Regulated ubiquitin-mediated protein turnover is one of the primary mechanisms governing passage through the cell cycle (Deshaies 1997). The central cell cycle regulator is the cyclin-dependent protein kinase (CDK; other organisms express multiple CDK variants), which is $\mathrm{Cdc28}$ in budding yeast. From a regulatory perspective, the cell cycle can be regarded as a sequence of timed regulatory subunit el iminations and replacements in the Cdc28 kinase complex; these regulatory subunits are the cyclins, which are positive regulatory subunits, and the CDK inhibitors (CKIs). In all cases examined, proteol ysis by the ubiquitin-proteasome pathway is the mechanism of subunit elimination. Additional proteins that must also be destroyed at specific times for cell cycle progression, for instance, Pds1 in yeast, are al so degraded by this pathway by mechanisms regulated, directly or indirectly, by CDKs (Deshaies 1997) .

In $\mathrm{S}$. cerevisiae, the $\mathrm{G}_{1}-\mathrm{S}$ transition is controlled by the destruction of a specific CKI, the Sicl protein (Schwob et al. 1994). Two B-type cyclins, Clb5 and Clb6, appear in $\mathrm{G}_{1}$ and associate with $\mathrm{Cdc} 28$; these $\mathrm{Cdc} 28$ complexes are important for entry into S phase but are inactive through earlier parts of $\mathrm{G}_{1}$ because they are bound tightly to Sicl, which accumulates shortly after mitosis. Sicl then is phosphorylated by the $\mathrm{G}_{1}$ cyclinCdc28 kinase (Verma et al. 1997). Phosphorylation of Sicl triggers its destruction by a specific E2/E3 ubiquitination complex (Fig. 3) (Feldman et al. 1997; Skowyra et al . 1997). The E2 in this case is Cdc34, whereas the E3 is a complex of three polypeptides, Skp1, Cdc53, and Cdc4, which is called SCFCdc4. Substrate binding to SCF ${ }^{\text {dc } 4}$ appears to be mediated by a series of WD-40 repeats in Cdc4, whereas a sequence motif in C dc4 called the F-box is necessary for binding to Skpl (Bai et al. 1996). Skp1 hel ps tether Cdc4 to Cdc53, the latter providing the link between the substrate binding subunits of the E3 complex and Cdc34 (Willems et al. 1996). Interestingly, Cdc53 and Skpl are part of several distinct E3 compl exes. What is common among the variable subunits in these complexes is the presence of the F-box motif and multiple copies of either WD-40 or leucinerich repeats (LRRs), which are both common proteinprotein interaction elements. For instance, a complex formed by Skp1, Cdc53, and Grr1, the latter an F-box and LRR-containing protein, appears to target the CInl and CIn2 $\mathrm{G}_{1}$ cyclins for degradation (Skowyra et al. 1997).

In addition to ubiquitinating Sic1, the C dc34/SCFCdc4 complex is also responsible for modification of Farl, a $\mathrm{CKI}$ required specifically to arrest the cell cycle in $\mathrm{G}_{1}$ in response to mating pheromones (Henchoz et al. 1997), and Cdc6, a protein essential for formation of prereplicative complexes (pre-RCs) at origins of DN A replication (Drury et al. 1997). Regulation of pre-RC assembly is important for making initiation of DNA replication dependent on passage through mitosis and in limiting replication to once per cell cycle. Cdc6 is normally present at high levels only during the $G_{1}$ phase of the cell cycle. This is attributable to cell cycle-regulated transcription of the CDC 6 gene and to Cdc34/SC F ${ }^{\text {dc4 }}$-dependent degradation of the $C$ dc 6 protein. Degradation of orthol ogs of Cdc6 in other species is likely to depend on ubiquitination by the equivalent of $\mathrm{Cdc} 34 / \mathrm{SCF}^{\mathrm{Cdc} 4}$ (Kominami and Toda 1997).

This brings us back, finally, to the data of Lammer et al. (1998) on Rub1 modification of Cdc53. First, it must be admitted that the rubl $\Delta$ mutant, as well as enr $2 \Delta$, uba $3 \Delta$, and ubc $12 \Delta$ strains, is distressingly healthy to anyone who would like to believe the Rubl pathway is playing a key regulatory role in the cell cycle. It is interesting, however, is that when mutations in CDC 34, CDC 4, CDC 53, or SKP1 are combined with the rub1 $\Delta$ or enr2 $\Delta$ mutations, the growth and cell cycle defects associated with the former group of mutations are enhanced significantly. These genetic interactions appear to be specific because combining the enr $2 \Delta$ mutation with mutations in other components of the ubiquitin system or cell cycle machinery (including grrl $\Delta$ ) does not cause comparable growth defects. Overproduction of Cdc34 or Cdc53 (but not, for reasons unknown, Cdc4) al so sensitizes cells to loss of the Rubl modification pathway. That these defects result from the absence of Rubl con- 
jugation to $\mathrm{Cdc53}$ is suggested by the identification of a functional $\mathrm{Cdc} 53$ carboxy-terminal truncation that renders the protein resistant to Rubl modification and at the same time makes cells sensitive to mutation of CDC 34. M oreover, overexpression of Cdc4 or Cdc34 inhibits the growth of cells bearing this truncated cdc53 allele but not cells carrying wild-type CDC53. A significant fraction of Cdc53 is modified by Rubl in logarithmically growing wild-type yeast cultures, and this fracti on increases substantially with overproduction of C dc4 or Cdc34. Finally, it is interesting to note that mutations in SKP1 al so prevent Rubl linkage to Cdc53. This could be attributable either to structural alteration of Cdc53/ Skpl-containing target complexes or to Skpl involvement in a Rubl-protein conjugation pathway.

What exactly are the consequences of Rubl modification? Attachment of Rub1 to Cdc53 might affect the partitioning of $\mathrm{Cdc53}$ among different Cdc53-containing E3 complexes, and/or it may change the activity of the Cdc34/SCF complex toward specific substrates. Lammer et al . (1998) suggest that Cdc53 metabolic stability is not affected because both modified and unmodified forms of the protein are degraded fairly slowly. In their pulsechase experiments, it does appear that the Rub1-Cdc53 conjugate might persist slightly longer than the free form (C dc53 turnover was not tested in a rub1 mutant). It will be important to determine whether NEDD 8 and other Rubl orthol ogs also modify cullins from other organisms (cullins comprise a well-conserved family of proteins related to $\mathrm{Cdc53).}$

M any of the components of Cdc34/SCF complexes appear to be widely conserved, and reports of new links between SCFs and the regulation of cell proliferation and development are now appearing in many quarters. In Caenorhabditis elegans, the cullin cul-1 is required for developmentally programmed transitions from $G_{1}$ to $G_{0}$ or to apoptotic pathways, so null mutants display a general hyperplasia; these mutants also appear to be abnormal in G-to-S phase regulation (Kipreos et al. 1996). Estelle and colleagues described a plant $F$ box-containing protein, TIR1, that is also critical for response to auxin and functions in the same response pathway as AXR 1 Ruegger et al . 1998). Drosophila limb patterning requires a protein called Slimb, an F-box protein related to Cdc4 that is important for processing or degradation of transcription factors regulated by the Wingless and Hedgehog signaling pathways (Jiang and Struhl 1998). In the fission yeast S. pombe, a protein called popl is critical for ubiquitination and degradation of cdc18; popl is closed related to Cdc4 of S. cerevisiae, and cdc18 is the likely ortholog of Cdc6 (Kominami and Toda 1997). Finally, mammalian cells contain a ubiquitination complex very similar to Cdc34/SCF (Lisztwan et al. 1998). This complex includes Cdc34, Skp1, CUL-1, and Skp2, with the latter two being the apparent counterparts of yeast Cdc53 and Grr1, respectively. Whether the cullins in any of these complexes are subject to Rub1/NEDD8 modification remains to be tested, but it is notable that a very prominent N EDD8-protein conjugate of 90-97 kD was observed in human cell extracts by Kamitani et al.
(1997), which would be compatible with a CUL-1 conjugate.

There are many obvious mechanistic and functional questions about Rub1/N EDD8 modification of proteins, but perhaps the most pressing concerns the apparent role of Rub1-Cdc53 conjugation in cell cycle regulation. To get at this issue, it is necessary first to consider the very different consequences of mutations in regulators of the $\mathrm{G}_{1} / \mathrm{S}$ transition in $\mathrm{S}$. cerevisiae versus other organisms. The reason this is likely to be relevant is the recent discovery (see Lammer et al. 1998) that the ts41 hamster cell line (Handeli and Weintraub 1992) harbors a temperature-sensitive allele of SMC1, a gene that is nearly identical to human APP-BP1, which encodes a subunit of the N EDD8-activating enzyme (see above). When ts41 cells are shifted to nonpermissive temperature, they traverse multiple $\mathrm{S}$ phases without intervening mitoses. This block to cell proliferation is in sharp contrast to the lack of obvious growth defect in yeast enr $2 \Delta$ cells. Instead, the ts41 defect is much more reminiscent of $\mathrm{S}$. pombe mutants with defects in $\mathrm{G}_{1} / \mathrm{S}$ regulators, which also uncouple the coordination of DNA replication and mitosis. Mutation of pop1, the Cdc4-like protein (see above), results in both accumulation of cdc18, and rereplication of the genome (Kominami and Toda 1997). Polyploidization is also observed in wild-type strains overproducing cdc18 (Brown et al. 1997). In contrast, constitutive overexpression of either wild-type or stable Cdc6 in budding yeast does not induce rereplication, suggesting the existence of a redundant mechanism for prevention of more than one $S$ phase in this organism (Drury et al. 1997). Interestingly, both Cdc6 and cdc18 bind tightly to CDK-cyclin B complexes, and Cdc6 inhibits kinase activity (Elsasser et al. 1996; Brown et al. 1997).

It is possible that the apparent redundancies in C dc34$\mathrm{SCF}^{\mathrm{Cdc} 4}$ regulation and in prevention of rereplication in budding yeast are not coincidental. For instance, the Rub1 modification of Cdc53 may be particularly important for $\mathrm{SCF}^{\mathrm{Cdc} 4}$ to ubiquitinate $\mathrm{Cdc} 6$ rather than Sicl, but because Cdc6 degradation is not essential for cell cycle progression in S. cerevisiae, deletion of the Rubl pathway is without major effect. In cells without Rubl and defective for $\mathrm{Cdc} 34 / \mathrm{SCF}^{\mathrm{Cdc} 4}$, the combined accumulation of $\mathrm{Cdc} 6$ and $\mathrm{Sic} 1$ may reduce $\mathrm{Clb}-\mathrm{Cdc} 28$ activity below that required to drive cells into $S$ phase (Farl accumulation, by inhibiting $\mathrm{Cln}-\mathrm{Cdc} 28$, may contribute indirectly as well). It is also conceivable that Rub1Cdc53 conjugation specifically enhances $C$ In ubi quitination by Cdc34/SCF. CIn degradation al so is not essential for cell cycle progression (Lanker et al. 1996), but failure to eliminate the CIns may sufficiently inhibit reloading of $\mathrm{Cdc} 28$ with $\mathrm{Clb}$ subunits to block the $\mathrm{G}_{1}$-to-S phase transition in cells also compromised for Cdc34/SCFCdc4. Perhaps a simpler idea is that Rubl modification of Cdc53 enhances Cdc34/SCF ${ }^{\text {Cdc4 }}$ activity toward all substrates. When loss of the Rubl pathway is combined with mutations or manipulations that further compromise Cdc34/SCF ${ }^{\mathrm{Cdc} 4}$ assembly or activity, the activity of the compl ex may drop below some threshold required for viability. A nother possibility is that Rubl conjugation to 
Cdc53 functions in a checkpoint pathway that ensures the proper temporal coupling of mitosis and DNA replication. In otherwise wild-type yeast cells grown under most conditions, no obvious growth defect would be expected from loss of the Rubl-dependent checkpoint.

\section{Future directions}

Clearly, these new data on Rubl and NEDD8 conjugation raise more questions than they answer. The dichotomy between the consequences of defects in this pathway in budding yeast versus plants and mammals is especially vexing. Whether there are fundamental paral lels between Rub1/N EDD8 and Smt3/SU M O-1 conjugation in cell cycle control is another question worth considering. An additional interesting analogy, discussed by Lammer et al. (1998), involves the human cullin CUL-2, which forms a complex with the von Hippel-Lindau tumor suppressor product, elongin $B$, and elongin $C$ (Pause et al. 1997). Elongin $C$ has similarity to Skpl, whereas elongin B is a Ubl. Perhaps Rubl and elongin B have similar functions in their respective heteromeric complexes. It will be useful to identify additional substrates for Rub1/NEDD8, which may, among other things, illuminate the apparent participation of this pathway in transduction of signals from the cell surface, which is suggested by APP-BP1/SMC 1 binding to plasma membrane APP in humans, and by the importance of $A X R 1$ to the auxin reponse in plants. At the current pace of research in the cell cycle and ubiquitin fields, we should not have long to wait for insights into these issues.

\section{Acknowledgments}

I thank Vincent Chau, M ark Estelle, and Stefan Jentsch for sharing data prior to publication, and Ray Deshaies, Jeff Laney, and Cecile Pickart for many helpful comments on the manuscript. Work in my laboratory on the ubiquitin system is supported by N ational Institutes of Health grants GM 46904 and GM 53756.

\section{References}

Bai, C., P. Sen, K. Hofmann, L. Ma, M. Goebl, J.W. Harper, and S.J. Elledge. 1996. SKP1 connects cell cycle regulators to the ubiquitin proteolysis machinery through a novel motif, the F-box. Cell 86: 263-274.

Brown, G.W., P.V. Jallepalli, B.J. Huneycutt, and T.J. Kelly. 1997. Interaction of the $S$ phase regulator cdc18 with cyclindependent kinase in fission yeast. Proc. Natl. Acad. Sci. 94: 6142-6147.

Burch, T.J. and A.L. Haas. 1994. Site-directed mutagenesis of ubiquitin. Differential roles for arginine in the interaction with ubiquitin-activating enzyme. Biochemistry 33: 73007307.

Chen, P., P. Johnson, T. Sommer, S. Jentsch, and M. Hochstrasser. 1993. Multiple ubiquitin-conjugating enzymes participate in the in vivo degradation of the yeast MAT $\alpha 2$ repressor. Cell 74: 357-369.

Chow, N., J.R. Korenberg, X.-N . Chen, and R. N eve. 1996. APP$\mathrm{BP} 1$, a novel protein that binds to the carboxy-terminal re gion of the amyloid precursor protein. J. Biol. Chem. 271: 11339-11346.
Deshaies, R.J. 1997. Phosphorylation and proteolysis: partners in the regulation of cell division in budding yeast. Curr. Opin. Genet. Dev. 7: 7-16.

Desterro, J.M., J. Thomson, and R.T. Hay. 1997. Ubch9 conjugates SU MO but not ubiquitin. FEBS Lett. 417: 297-300.

Drury, L.S., G. Perkins, and J.F. Diffley. 1997. The Cdc4/34/53 pathway targets Cdc6p for proteolysis in budding yeast. EMBO J. 16: 5966-5976.

Elsasser, S., F. Lou, B. Wang, J.L. Campbell, and A. Jong. 1996. Interaction between yeast Cdc6 protein and B-type cyclin/ Cdc28 kinases. Mol. Biol. Cell 7: 1723-1735.

Feldman, R.M., C.C. Correll, K.B. Kaplan, and R.J. Deshaies. 1997. A complex of Cdc4p, Skplp, and Cdc53p/cullin catalyzes ubiquitination of the phosphorylated CDK inhibitor Sic1p. Cell 91: 221-230.

Haas, A.L. and T.J. Siepmann. 1997. Pathways of ubiquitin conjugation. FASEB J. 11: 1257-1268.

Handeli, S. and H. Weintraub. 1992. The ts41 mutation in Chinese hamster cells leads to successive $S$ phases in the $a b-$ sence of intervening G2, M, and G1. Cell 71: 599-611.

Henchoz, S., Y. Chi, B. Catarin, I. Herskowitz, R.J. Deshaies, and M. Peter. 1997. Phosphorylation and ubiquitin-dependent degradation of the cyclin-dependent kinase inhibitor Far1p in budding yeast. Genes \& Dev. 11: 3046-3060.

Hochstrasser, M. 1996. Ubiquitin-dependent protein degradation. Annu. Rev. Genet. 30: 405-439.

Jiang, J. and G. Struhl. 1998. Regulation of the hedgehog and wingless signalling pathways by the F-box/WD40-repeat protein Slimb. Nature 391: 493-496.

Johnson, E.S. and G. Blobel. 1997. Ubc9p is the conjugating enzyme for the ubiquitin-like protein Smt3p. J. Biol. Chem. 272: 26799-26802.

Johnson, E.S., I. Schwienhorst, R.J. Dohmen, and G. Blobel. 1997. The ubiquitin-like protein Smt3p is activated for conjugation to other proteins by an Aoslp/U ba2p heterodimer. EMBO J. 16: 5509-5519.

Johnson, P.R. and M. Hochstrasser. 1997. SUMO-1: ubiquitin gains weight. Trends Cell Biol. 7: 408-413.

Kamitani, T., K. Kito, H.P. N guyen, and E.T. Yeh. 1997. Characterization of NEDD8, a developmentally down-regulated ubiquitin-like protein. J. Biol. Chem. 272: 28557-28562.

Kipreos, E.T., L.E. Lander, J.P. Wing, W.W. He, and E.M. Hedgecock. 1996. cul-1 is required for cell cycle exit in C. elegans and identifies a novel gene family. Cell 85: 829-839.

Kominami, K. and T. Toda. 1997. Fission yeast WD-repeat protein pop1 regulates genome ploidy through ubiquitin-proteasome-mediated degradation of the CDK inhibitor Rum 1 and the S-phase initiator Cdc18. Genes \& Dev. 11: 1548-1560.

Kumar, S., Y. Y oshida, and M. N oda. 1993. Cloning of a CDN A which encodes a novel ubiquitin-like protein. Biochem. Biophy. Res. Comm. 195: 393-399.

Lammer, D., N. Mathias, J.M. Laplaza, W. Jiang, Y. Liu, J. Callis, M. Goebl, and M. Estelle. 1998. Modification of yeast Cdc53p by the ubiquitin-related protein Rublp affects function of the SCF ${ }^{\mathrm{Cdc} 4}$ complex. Genes \& Dev. (this issue).

Lanker, S., M.H. Valdivieso, and C. Wittenberg. 1996. Rapid degradation of the $\mathrm{G} 1$ cyclin CIn induced by Cdk-dependent phosphorylation. Science 271: 1597-1601.

Leyser, H.M.O., C. Lincoln, C. Timpte, D. Lammer, J. Turner, and M. Estelle. 1993. The auxin-resistance gene AXR1 of Arabidopsis encodes a protein related to ubiquitin-activating enzyme E1. Nature 364: 161-164.

Liakopoulos, D., G. Doenges, K. M atuschewski, and S. Jentsch. 1998. A novel protein modification pathway related to the ubiquitin system. EMBO J. (in press).

Lisztwan, J., A. Marti, H. Sutterlüty, M. Gstaiger, C. Wirbe- 
Iauer, and W. Krek. 1998. Association of human CUL-1 and ubiquitin-conjugating enzyme CDC34 with the F-box protein p45 ${ }^{\text {SKP2: }}$ Evidence for evolutionary conservation in the subunit composition of the CDC34-SCF pathway. EMBO J. 17: 368-383.

Pause, A., S. Lee, R.A. Worrell, D.Y.T. Chen, W.H. Burgess, W.M. Linehan, and R.D. Klausner. 1997. The von HippelLindau tumor suppressor gene product forms a stable complex with human CUL-2, a member of the Cdc53 family of proteins. Proc. Natl. Acad. Sci. 94: 2156-2161.

Pickart, C.M. 1997. Targeting of substrates to the $26 \mathrm{~S}$ proteasome. FASEB J. 11: 1055-1066.

Ruegger, M., E. Dewey, W.M. Gray, L. Hobbie, J. Turner, and M. Estelle. 1998. The TIR1 protein of Arabidopsis functions in auxin response and is related to human SKP2 and yeast Grrlp. Genes \& Dev. 12: 198-207.

Scheffner, M., U. Nuber, and J.M. Huibregtse. 1995. Protein ubiquitination involving an E1-E2-E3 enzyme ubiquitin thioester cascade. Nature 373: 81-83.

Schwarz, S.E., K.D.L. Matuschewski, M. Scheffner, and S. Jentsch. 1998. The ubiquitin-like proteins SMT3 and SUM O-1 are conjugated by the UBC 9 E2 enzyme. Proc. Natl. Acad. Sci. 95: 560-564.

Schwob, E., T. Böhm, M.D. Mendenhal I, and K. N asmyth. 1994. The B-type cyclin kinase inhibitor $\mathrm{p} 40^{\mathrm{SIC1}}$ controls the $\mathrm{G} 1$ to S transition in S. cerevisiae. Cell 79: 233-244.

Skowyra, D., K.L. Craig, M. Tyers, S.J. Elledge, and J.W. Harper. 1997. F-box proteins are receptors that recruit phosphorylated substrates to the SCF ubiquitin-ligase complex. Cell 91: 209-219.

Verma, R., R. Annan, M. Huddleston, S. Carr, G. Reynard, and R.J. Deshaies. 1997. Phosphorylation of Siclp by G1 cyclin/ $\mathrm{Cdk}$ is required for its degradation and entry into $\mathrm{S}$ phase. Science 278: 455-460.

Wilkinson, K.D. 1995. Roles of ubiquitinylation in proteolysis and cellular regulation. Annu. Rev. Nutr. 15: 161-189.

Willems, A.R., S. Lanker, E.E. Patton, K.L. Craig, T.F. N ason, R. Kobayashi, C. Wittenberg, and M. Tyers. 1996. Cdc53 targets phosphorylated G1 cyclins for degradation by the ubiquitin proteolytic pathway. Cell 86: 453-463. 


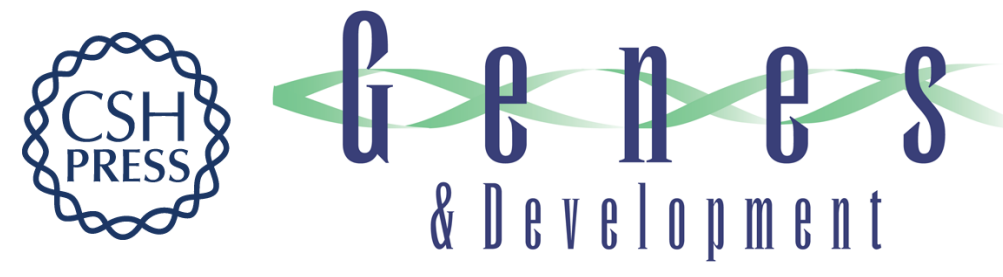

\section{There's the Rub: a novel ubiquitin-like modification linked to cell cycle regulation}

Mark Hochstrasser

Genes Dev. 1998, 12:

References This article cites 35 articles, 15 of which can be accessed free at:

http://genesdev.cshlp.org/content/12/7/901.full.html\#ref-list-1

License

Email Alerting Receive free email alerts when new articles cite this article - sign up in the box at the top Service right corner of the article or click here.

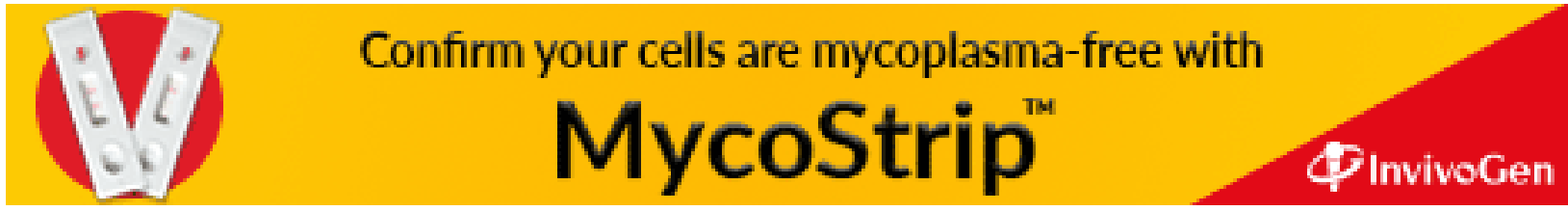

